Breda Cigoj-Leben

Ljubljana

\title{
ANDRÉ GIDE ET LES FLEURS DU MAL DE BAUDELAIRE
}

André Gide connut à fond le charme de la poésie vers 1885 , à l'âge de seize ans, quand on lui permit de fouiller seul dans la bibliothèque de son père. A cette époque il tenait la poésie pour «la fleur et l'aboutissement de la vie» $(J . I I, 1491)$. Ce fut Victor Hugo dont il apprit par cœur nombre de pièces, ce fut surtout Henri Heine, lu d'abord dans la traduction et bientôt dans le texte, qui le boulversèrent profondément:

Je me revois, étendu sur le tapis, à l'étrusque, au pied de la petite bibliothèque ouverte, en ce printemps de ma seizième année, tremblant à découvrir, à sentir s'éveiller et répondre à l'appel d'Henri Heine l'abondant printemps de mon cœur( Ibid., 482).

Nous ne savons pas exactement quand il fit la première connaissance de Baudelaire. En tout cas, Baudelaire était un des auteurs que Gide lisait déjà «avec les ambitions révélées» $(A W, 223)$ et en compagnie de sa cousine Emmanuèle - Madeleine, peut-être en 1888, pendant ses vacances à La Roque. Dans son Subjectif, cahier où il notait ses lectures entre 1889 et 1893, la première lecture de Baudelaire est signalée le 17 novembre 1889 , mais l'ouvrage lu n'est pas nommé. ${ }^{3}$ Il s'agit sans doute d'une lecture des Fleurs du Mal ou du moins d'un certain nombre de pièces de ce recueil poétique, car de nombreux vers de Baudelaire cités dans André Walter qui fut achevé l'été 1890 témoignent que Les Fleurs du Mal étaient assez familières à Gide quand il rédigeait sa première cuvre.

Les vers de Baudelaire que Gide cite dans André Walter sont choisis de manière à souligner les sentiments du protagoniste qui d'ailleurs représente assez fidèlement le jeune Gide. Ce qui est significatif dans le choix de ces vers, c'est l'intérêt que montre Gide pour les motifs noctur-

1 André Gide: Journal 1939-1949. Souvenirs, Paris, Gallimard («Bibliothèque de la Pléiade»), 1945.

2 André Gide: André Walter. Cahiers et Poésies, Éd. définitive, Paris, Les Oeuvres représentatives, 1930.

3 Jacques Cotman: «Le Subjectif, ou les lectures d'André Walter», dans: Cahiers André Gide 1, Paris, Gallimard, 1969, p. 59. 
nes. Voici le salon plongé dans le crépuscule, où la famille est rassemblée; André Walter récite l'Expiation de Victor Hugo. Tout à coup il sent dans l'ombre le regard d'Emmanuèle fixé sur lui, et ce regard lui rappelle un vers du Balcon de Baudelaire: «Et mes yeux dans le noir devinaient tes prunelles» $(A W, 56)$. Vu le contexte, le ton de ce vers devient pourtant différent de celui dans le poème de Baudelaire. Dans l'ombre recueillie du salon familial à $L a$ Roque, les yeux rencontrés par Gide sont les yeux purs de l'angélique Emmanuèle, tandis que dans le poème de Baudelaire les yeux que le poète contemple dans une chaude soirée d'été sur le balcon d'un hôtel parisien sont ceux de la «maîtresse des maîtresses» dont il boit le souffle qui contient et la douceur et le poison.

Comme André Walter, Emmanuèle aussi aime citer les poètes parce que leurs vers lui rappellent tant de choses: les lectures faites avec André, les émois communs. Les affinités entre les deux jeunes gens sont si profondes que souvent ils prononcent à la fois les mêmes mots:

Nous prévoyons venir les phrases; nous nous les dérobions avant qu'elles ne fussent dites, sur nos lèvres prêtes à parler, - et l'attente seule d'une pensée chez l'autre la faisait parfois naître chez les deux la même $(A W, 67)$.

Cette pensée, née chez les deux à la fois, s'exprime très souvent par un vers de Baudelaire. Dans les soirs d'été le vers suivant leur revient sur les lèvres:

Ce soir la lune rêve avec plus de paresse... $(A W, 68)$.

Le vers cité est emprunté à la Tristesse de la lune de Baudelaire, poème qui correspond très bien à la mélancolie du jeune et amoureux Walter et à ses aspirations du «poète pieux, ennemi du sommeil», comme dit Baudelaire.

Un autre vers de Baudelaire est cité à la même occasion:

Comme tu me plairais, ô nuit! sans tes étoiles...

vers trouvé dans l'Obsession de Baudelaire, sonnet qui par son évocation du néant - «Car je cherche le vide, et le noir, et le nu!» - correspond moins à l'état d'âme d'André et Emmanuèle. Le couple amoureux ne se soucie pas, d'ailleurs, d'achever la citation: «Mais le vers inachevé retombait de nos lèvres lassées, pour laisser chanter le regard qui mieux disait nos tendresses vivaces et toujours désireuses» $(A W, 68)$.

Il y a enfin un vers de Baudelaire tellement cher aux deux amants qu'ils le répètent à toute occasion. Il s'agit du célèbre dernier vers du beau sonnet Recueillement. André commence: «Entends! ma chère...», et Emmanuèle replique: «Entends la douce nuit qui marche» $(A W, 67)$. En récitant ce vers le jeune couple se trouve devant la campagne tranquille que peu à peu enveloppe la nuit; le milieu est donc assez différent de celui du poème de Baudelaire qui, seul, au milieu de la tumultueuse 
ville, voyant le soleil se coucher derrière les ponts de la Seine, s'adresse à sa douleur:

[...] Vois se pencher les défuntes années,

Sur les balcons du ciel, en robes surannées;

Surgir du fond des eaux le Regret souriant;

Le Soleil moribond s'endormir sous une arche.

Et comme un long linceul traînant à l'Orient,

Entends, ma chère, entends la douce nuit qui marche.

Après le mariage d'Emmanuèle, Walter solitaire contemple encore la campagne:

Les ombres ont grandi et se sont étendues. Les grands rayons ont ébloui la plaine. Le soleil s'est couché - les chants du soir - comme autrefois. Autrefois... notre âme s'illuminait aux mutuels reflets de notre extase; j'écoutais en toi l'écho de mes adorations silencieuses $(A W, 112)$.

Le jeune homme abandonné se souvient comment naguère, la nuit venue, il avait commencé: «Entends; ma chère ...», pour se sentir répondre: «Entends la douce nuit qui marche». Et, triste, il soupire: «- refrain des littératures apprises, qui remonte du passé avec les souvenirs» $(A W, 113)$. C'est encore après la mort d'Emmanuèle et quand Walter sombre dans la folie que ce vers lui revient comme une obsession: «[...] mais quand tout se tait, l'on écoute - la nuit, la grande nuit..., Entends, ma chère $\ldots \gg(A W, 220)$.

$$
* * *
$$

Depuis André Walter et le Subjectif Gide complète progressivement sa connaissance de la poésie de Baudelaire. Ainsi, dans une lettre non datée qui a dû être écrite entre 1894 et 1898, écrit-il à Paul Valéry: « Je lis de Baudelaire bien des pièces que je ne connaissais pas» ; ${ }^{4}$ il cite des vers du Serpent qui danse et de L'Impréou.

Le 14 juin 1905 Baudelaire est mentionné pour la première fois dans le Journal de Gide: »Repris Baudelaire avec le plus vif plaisir» $\left(J .1,{ }^{5}\right.$ 166). Dans son âge mûr et dans sa vieillesse Gide revient régulièrement à Baudelaire dans son Journal, quelquefois aussi dans ses lettres et dans ses récits; mais il rédige surtout deux écrits importants consacrés au grand poète français du XIXe siècle: en 1910 l'article «Baudelaire et M. Faguet» et en 1917 une «Préface» aux Fleurs du Mal. II assigne enfin à Baudelaire une large place dans son Anthologie de la poésie française qui paraît en 1949.

4 «André Gide et Paul Valéry. Dix lettres inédites», dans: Bulletin des amis d'André Gide, vol. IV, No 29, Janvier 1976, p. 6.

s André Gide: Journal 1889-1939, Paris, Gallimard («Bibliothèque de la Pléiade»), 1951. 
Baudelaire devient si familier à Gide que lorsque, au seuil de la vieillesse, pour exercer sa mémoire, celui-ci se redit inlassablement «de larges pans de vers» de ses poètes préférés, parmi ces poètes se trouve tout naturellement Baudelaire $(J . I I, 12)$; et Gide se sent tout penaud quand il ne réussit pas à se remémorer complètement Le Crépuscule du Matin $(J . I I, 137)$.

Dans son âge mûr Gide continue à chercher dans la poésie de Baudelaire les images et les émotions qui lui paraissent correspondre à ses propres situations ou états d'âme, ou à ceux des personnages de ses récits. Mais l'application des vers de Baudelaire à ces situations, limitée dans André $W$ alter à un choix des vers plutôt restreint, se fait maintenant plus large. Ainsi trouvons-nous dans La Porte étroite (1909) un passage qui rappelle des passages déjà cités d'André $W$ alter mais dont le ton est assez différent. Il s'agit de l'épisode ou Juliette, jalouse, veut savoir quels vers Jérome avait récités le jour précédent à Alissa. Jérome commence «d'assez mauvaise grâce»: «Bientôt nous plongerons dans les froides ténèbres»; mais à sa surprise Juliette l'interrompt continuant «d'une voix tremblante et changée»: «Adieu, vive clarté de nos étés trop courts». ${ }^{B}$

A une autre occasion, il paraît à Gide de pouvoir caractériser par un vers de Baudelaire une de ses propres œuvres. Sa sotie Les Caves $d u$ Vatican lui paraît au moment de la publication (1914) si froide et peu spirituelle qu'il croit pouvoir mettre sur la couverture d'une nouvelle édition en épigraphe le vers de Baudelaire:

Je hais la passion et l'esprit me fait mal. ${ }^{\top}$

Il y a une situation particulière qui permet à Gide de retrouver très souvent ses impressions dans les vers de Baudelaire: c'est quand il voyage dans des pays exotiques. Significatives sont dans ce domaine les comparaisons que suggèrent à Gide la vie, les êtres humains et la nature exotique lors de son voyage au Congo (en 1925/26). De Fort Archambault il écrit à Roger Martin du Gard d'y goûter ce que Baudelaire appelait «la féconde paresse». 8 L'expression se trouve effectivement dans La Cheoelure des Fleurs du Mal. A un autre moment du même voyage, un jeune homme de couleur, fils du chef d'un village, «beau, svelte, élégant» $(J . I I, 796)$, le fait penser - au moyen d'une étrange confusion des sexes - à la Sisina, la belle aventurière que Baudelaire, dans son sonnet homonyme; compare à la Diane-chasseresse.

Moins déroutant est un autre rapprochement fait à propos des végétaux des contrées équatoriales qui tendent vers «une forme symétrique

${ }^{6}$ André Gide: Romans, récits et soties, ceuvres lyriques, Paris, Gallimard ( Bibliothèque de la Pléiade»), 1958, p. 517.

${ }^{7}$ Le vers cité se trouve dans le Sonnet d'Automne de Baudelaire.

8 André Gide - Roger Martin du Gard: Correspondance, Paris, Gallimard. 1968, t. I, p. 280. 
et comme cristalline, insoupçonnée dans nos pays du Nord où Baudelaire peut parler du 'végétal irrégulier'» $(J . I I, 829)$. Effectivement, dans son Rêpe parisien, décrivant un étrange paysage qui n'est que métal, marbre et eau et sur lequel plane «un silence d'éternité», Baudelaire parle de l'absence du «végétal irrégulier», pour souligner l'absence, de ce monde aux formes géométriques, à «l'enivrante monotonie», de tout signe d'une vie organique:

\section{J'avais banni de se spectacle \\ Le végétal irrégulier.}

Si pour Baudelaire la symétrie signifie l'absence de la vie végétale, Gide, tout au contraire, voit dans les plantes exotiques aux formes symétriques un regain de la vitalité et de la puissance de la nature.

Baudelaire n'aime pas la nature, ce qui explique pourquoi la vue de son «terrible paysage», nu et sans le moindre signe de vie, le «ravit». On sait d'ailleurs avec quel mépris il parle, dáns une de ses lettres, des plantes, ces «légumes sanctifiés» par les romantiques, ajoutant: "J'ai même toujours pensé qu'il y avait dans la Nature, florissante et rajeunie, quelque chose d'impudent et d'affligeant.$^{9} \mathrm{Et}$ pourtant, Gide qui aime passionnément la nature - celle de l'Afrique équatoriale et l'autre, plus modeste, du Midi et du Nord de la France - qui ne s'intéresse pas aux animaux et aux plantes seulement en homme de lettres mais aussi en entomologiste et en botaniste et qui éprouve de «brusques attendrissements devant la beauté de certaines fleurs» $(J . I, 1239)$, sait trouver dans Les Fleurs du $\mathrm{Mal}$ un des rares exemples où il voit exprimée cette même émotion. Gide se demande un jour si les formes de la vie, qui dans les animaux et les plantes sont si charmantes par rapport à l'homme, travaillent aussi à la joie, à l'intime satisfaction des créatures qui les possèdent. On croit trop souvent que le parfum de la fleur et la suavité de ses couleurs n'ont «d'autre fin que de caresser l'œil ou les narines de l'homme» $(J . I, 1240)$. Gide trouve que Baudelaire aussi fut coupable de cet «anthropocentrisme sournois d'une charmante naïveté» quand il écrivit les vers «exquis entre tous»:

Mainte fleur épanche à regret

Son parfum doux comme un secret

Dans les solitudes profondes.

Gide remarque d'ailleurs que ces vers - il les a tirés du poème Le Guignon - ne sont pas originaux mais traduisent «miraculeusement» deux vers célèbres du poète anglais Thomas Gray.

De même que la poésíe de Baudelaire jouait un rôle important dans le développement sentimental du protagoniste d'André Walter, œuvre de

๑ Charles Baudelaire: Correspondance,.Paris, Gallimard ( $«$ Bibliothèque de la Pléiade»), 1973, t. I, p. 248. 


\section{Linguistica $X V$}

jeunesse de Gide, de même qu'elle apparut, chargée d'émotion, au milieu de sa vie dans un épisode clef de La Porte étroite, de même elle confère une atmosphère exceptionnelle à certaines pages d'un de ses derniers récits, à sa Geneoiève (1936). Il s'agit de l'épisode où une belle jeune fille, la précoce et indolente juive Sara, récitant «d'une voix égale, riche mais extraordinairement douce et voilée» La Mort des Amants, ce «poème admirable» qu'elle aime tout particulièrement, donne à Geneviève, si positive et rationaliste par sa nature, «la soudaine révélation d'un autre monde dont le monde extérieur ne serait que le pâle et morne reflet».10 Pour compléter l'effet obtenu Sara récite encore L'Inoitation au voyage et Le Jet d'eau.

Gide trouve son propre moi dans les vers de Baudelaire de la manière la plus frappante en août de 1938. L'événement se produit peu de temps après la mort subite de sa femme; il se sent comme perdu, il ne s'intéresse à rien, il n'attend plus rien de la vie: «[...] depuis qu'Em. m'a quitté j'ai perdu goût à la vie $[. .]>.(J . I, 1309)$. Alors ses yeux tombent presque par hasard sur quelques vers d'un poème de Baudelaire qui lui paraissent inconnus, mais qui le frappent par leur extraordinaire ressemblance avec son état d'âme actuel:

Ce vers répondait si étrangement à mon état présent qu'il me semble que Baudelaire l'avait écrit tout particulièrement pour moi et pour cet instant précis de ma vie (lbid.).

Il s'agit des vers suivants:

Quand notre coeur a fait une fois sa vendange,

Vivre est un mal. C'est un secret de tous connu."11

La puissance incantatrice et la force consolatrice de ces vers proviennent pour Gide du fait qu'ils généralisent et invitent à appliquer à tout être humain ce qu'il a considéré comme une vérité profondément douloureuse connue de lui seul. ${ }^{2}$

\section{$* * *$}

Gide ne se limite pas à appliquer les vers de Baudelaire à ses situations personnelles, il fait aussi des réflexions critiques sur l'essence et la valeur de la poésie de Baudelaire. Ces réflexions, il les exprime surtout dans les deux écrits déjà mentionnés: l'article «Baudelaire et M. Faguet»13 et la «Préface» aux Fleurs du Mal.14 Gide prend pour le point de départ

10 André Gide: Romans, récits et soties ..., p. 1365.

1 Semper eadem dans Les Fleurs du Mal.

12 Sur cet événement voir aussi la lettre de Gide à Martin du Gard du 21 août 1928 (André Gide - Roger Martin du Gard: Correspondance, t. II, p. 148).

${ }_{13}$ La Noudelle Reoue Française, 1er Novembre 1910; l'article fut recueilli ensuite dans les Nouveaux Prétextes.

14 Charles Baudelaire: Les Fleurs du Mal, Paris, Editions Pelletan, R. Helleu, 1917 ; cette préface fut réimprimée dans les Incidences. 
de son article «Baudelaire et M. Faguet» un article de Faguet sur Baudelaire, paru dans la Reoue du 1er septembre 1910, bâclé à la hâte, plein de négligences, injuste, et tout de même important, vu le prestige du critique. Faguet y traite Baudelaire comme sun bon poète de second ordre», il y exprime sa surprise de voir que Baudelaire, quand une génération a passé et une autre est au milieu de sa course, n'a pas encore sombré dans l'oubli. Il lui reproche de n'avoir aucune idée neuve; ses sujets décèlent la banalité, il ne fait que répéter les lieux communs, et même en déployant ces lieux communs il est gauche, lourd, plat, il ne connaît pas la valeur de la forme. Gide oppose aux reproches de Faguet d'abord l'affirmation très juste qu'on ne fait pas la poésie avec des idées: «En art, où l'expression seule importe, les idées ne paraissent jeunes qu'un jour» (N.P.,15 140). Il constate ensuite, encore contre Faguet, que la seule explication possible du mystère de la survie de Baudelaire est précisément la forme: «[...] c'est à la perfection de sa forme que Baudelaire doit sa survie. L'artiste la doit-il jamais à rien d'autre?» $(N . P ., 141)$. Dans sa «Préface aux Fleurs du Mal» qui complète l'article «Baudelaire et M. Faguet» Gide répète que c'est la forme qui représente la «raison de l'œuvre d'art», le «secret de l'œuvre» (Inc., 16 166). Gide comprend d'ailleurs que parlant de la forme il ne pense pas à la même chose que Faguet. Pour Faguet la perfection de la forme signifie une «perfection toute latine», une perfection «rhétorique, logique, oratoire» et qui peut être expliquée $(N . P ., 142)$; à cette conception traditionnelle Gide oppose très heureusement sa propre conception de la perfection de la forme: c'est une «perfection plus cachée, musicale déjà» (Ibid.) et qui consiste dans «cette harmonie des contours et des sons où l'art du poète se joue» (Inc., 166).

Employant l'adjectif «musical» Gide ne le met pas en rapport seulement avec «la caresse fluide et le choc harmonieux des sonorités verbales» (N. P., 146) - qualités par lesquelles un vers peut plaire même à celui qui ne connaît pas une langue - mais aussi avec le choix et le rapprochement des expressions par lesquels le poète échappe à la logique et arrive à fixer «l'émotion essentiellement indéfinissable» (Ibid.). Il y a dans ces rapprochements, comme remarque Gide, une apparence d'imprécision, les termes semblent impropres à la première vue. Mais c'est précisément dans ce qu'il appelle le «laps entre l'image et l'idée» ${ }^{17}$ que «l'émotion poétique va pouvoir habiter» (N.P., 148). Baudelaire évite les lieux communs, les métaphores prévues, il est persuadé qu'une associa-

15 André Gide: Nouveaux Prétextes, Paris, Mercure de France, 1911.

${ }^{16}$ André Gide: Incidences, Paris, Gallimard, 1924.

12 Gide emploiera plus tard l'expression «écrasement poétique de la pensée», citant comme exemple des vers du Recueillement et de L'Amour du Mensonge de Baudelaire (André Gide: Divers, Paris, Gallimard, 1931, p. 79). Aujourd'hui on préfère parler de la manière synthétique de s'exprimer et du rythme intérieur de la poésie. 
tion des images et des mots est parfaite non point «lorsqu'elle peut servir toujours, mais au contraire quand elle ne peut servir qu'une fois» $(N . P$., 149). Pour illustrer cette affirmation Gide cite et explique les dernières trois strophes du poème de Baudelaire Mcesta et errabunda.

Une des conséquences de cette musicalité intérieure, de ces rapprochements inattendus des expressions est le fait qu'on ne peut pas remplacer un seul mot dans un vers de Baudelaire; si l'on essaie de le faire il arrive, comme remarque Gide, que «l'harmonie tout entière du vers et de la strophe, le son du poème entier parfois, n'est plus que celui d'une belle cloche fêlée» $(N: P ., 148)$. Il s'aperçoit aussi que c'est d'ordinaire dans les pièces brèves que la musicalité est le mieux réalisée. Plus tard, en 1934, il parlera de l'amour de Baudelaire pour le sonnet, précisément parce que cette forme restreinte interdit «l'inspiration successive» $(J . I, 1214)$. Il citera à cette ocasion des vers du beau sonnet $P$ arfum exotique.

La sobriété et la densité de l'expression baudelairienne font formuler à Faguet encore un reproche: Baudelaire n'a quasi aucune imagination. Gide se demande si «c'est bien essentiellement l'imagination qui fait le poète» $(N . P$., 150). Pour y répondre il se sert d'un aphorisme d'Oscar Wilde: «L'imagination imite, c'est l'esprit critique qui crée». Fort de cette constatation il affirme que Baudelaire était, avec Stendhal, »la plus admirable intelligence critique de son époque» $(N . P ., 151)$; mais il corrige aussitôt ce que cette affirmation, appliquée à un poète, semble contenir de paradoxal; il explique, appuyé encore sur Wilde, que le mot critique se rapporte ici moins à l'œuvre d'autrui qu'à soi-même. Wilde affirme, et Gide le répète, qu'il s'y agit de «ce délicat instinct de sélection avec lequel l'artiste crée la vie pour nous et lui donne une perfection momentanée» (N.P., 153). Celui qui ne possède pas ce «subtil tact d'omission» ne peut rien créer en art (Ibid.). ${ }^{18}$ C'est par son «immanent sens critique» que Baudelaire, selon Gide, se sépare du romantisme et, tout en gardant «la frémissante conscience de sa modernité», s'y oppose (Ibid.); ce qui veut dire en d'autres termes que Baudelaire est pour Gide un classique.

En 1921, dans ses Billets à Angèle, Gide parle des qualités de l'œuvre classique: «L'œuvre classique ne sera forte et belle qu'en raison de son romantisme dompté (Inc., 38). D’après ce que.Gide affirme dans un appendice aux Billets le classicisme et le romantisme ne représentent pas pour lui seulement deux mouvements historiques mais aussi et surtout deux tendances qui se manifestent à tout moment dans la lutte qui «existe à l'intérieur de chaque esprit»' (Inc., 217) et qui est indépendante de l'époque. Il complète sa définition du classicisme: «[...] l'œuvre d'art classique raconte le triomphe de l'ordre et de la mesure sur le romantisme intérieur».(Ibid.). L'ordre et la mesure ne peuvent pas être réalisés sans

18 Des termes plus usités pour désigner «ce subtil tact d'omission», «ce délicat instinct de sélection», qui créent l'œuvre d'art poétique, seraient: fantaisie créatrice, goût. 
«l'immanent sens critique», sans «le tact d'omission» qu'il avait déjà attribué à Baudelaire.

La conception gidienne du classicisme qui serait le «romantisme dompté» nous fait comprendre aussi l'usage que Gide fait des termes poète et artiste. Il lui arrive souvent de les mettre en opposition. Le terme poète se rapporte alors à l'auteur qui n'a pas su dompter ses sentiments, soumettre son romantisme, tandis que l'artiste est celui qui a su transformer ses sentiments et leur donner une forme. Gide avait noté cette distinction pour la première fois déjà en 1892 dans son Journal; mais comme il y faisait la confusion de l'art et de la morale - mot d'ailleurs très vague dans la pensée gidienne de cette époque - l'emploi des termes poète et artiste y restait ambigu: «Il faut que l'artiste supplante le poète. De la lutte entre les deux naît l'œuvre d'art» $(J . I ., 30)$. L'emploi des deux termes est tout à fait clair quand dans «Baudelaire et M. Faguet» Gide dit qu'il faut saluer en Baudelaire «autre chose et plus qu'un poète: le premier artiste en poésie» $(N . P ., 150)$.

Lorsque, dans les Billets à Angèle, Gide affirme que le classicisme tend vers la litote - «l'art d'exprimer le plus en disant le moins», «un art de pudeur et de modestie» (Inc., 40) - il pense aussi aux rapprochements synthétiques et suggestifs des mots dans la poésie de Baudelaire, car énumérant les auteurs français chez qui l'on sent «que la parole, tout en révélant l'émotion, ne la contient pas toute et que, une fois le mot prononcé, l'émotion qui le précédait, continue», il cite à côté de Racine et de Pascal précisément Baudelaire (Inc., 41).

C'est d'ailleurs dans certains vers de Baudelaire que Gide cherche en 1918 «la parfaite définition de l'œuvre d'art» (J. I., 664). Il s'agit de deux vers qui se répètent trois fois dans L'Invitation au voyage:

Là, tout n'est qu'ordre et beauté,

Luxe; calme et volupté.

Gide voudrait transformer un peu arbitrairement les mots de ces vers, qui se rapportent au rêve baudelairien de la splendide et indolente vie des pays orientaux, en autant de titres des successifs chapitres d'un traité d'esthétique. Dans cette interprétation très libre des deux vers de Baudelaire on peut apercevoir précisément les éléments du romantisme et du classicisme gidiens: d'un côté la volupté - «sensualité, charme adorable de la matière, attrait»; de l'autre côté le luxe - «abondance disciplinée», le calme - «tranquilisation du tumulte», l'ordre - «logique, disposition raisonable des parties» et la beauté - «ligne, élan, profil de l'œuvre» $(J . I, 664)$.

Il est intéressant de voir comment, arrivé aux intuitions esthétiques très justes, Gide juge l'un ou l'autre des poèmes de Baudelaire. Dans «Baudelaire et M. Faguet». il accuse Faguet de louer L'Homme et la mer et le quatrième Spleen qui seraient "deux des moins bonnes pièces du 
recueil» $(N . P ., 143)$. Si ce jugement est valable pour la première pièce mentionnée, le quatrième Spleen:

Quand le ciel bas et lourd pèse comme un couvercle

Sur l'esprit gémissant en proie aux longs ennuis...

où le poète a su exprimer avec une puissance extraordinaire son angoisse, certes ne mérite pas la critique sévère de Gide.

Faguet loue Don Juan aux Enfers comme tableau; Gide, sans se prononcer sux cet admirable poème, remarque que Barrès aurait voulu l'enlever du volume. Faguet admire le mouvement d'Une Charogne, Gide replique que du mouvement, il en trouverait davantage dans les Odes de J.-B. Rousseau et qu'une des plus grandes nouveautés de l'art baudelairien est précisément d'immobiliser ses images. Faguet parle «avec complaisance» de la pièce intitulée Confession. Gide lui en veut d'y critiquer deux vers qu'il n'a pas pris la peine de citer exactement, mais la pièce entière qui est assez belle, Gide la trouve «décevante» $(N . P ., 144)$.

Gide est plus juste envers Baudelaire quand il parle des poèmes qu'i] préfère et que Faguet précisément ne cite pas. Outre les trois très belles dernières strophes de Mosta et errabunda reproduites en entier, Gide nomme Le Balcon, L'Invitation au doyage, Le Crépuscule du Matin et Le Jet d'eau - quatre poèmes très beaux ou même parfaits. On peut hésiter un peu devant son choix de La Cheoelure qui, tout en contenant de beaux vers, n'est pas exempte de rhétorique: Gide fut attiré évidemment par son exotisme - c'est dans ce sens qu'il cite plus tard, lors de son séjour à Fort Archambault, «la féconde paresse» de La Chevelure.

$$
\text { *** * }
$$

L'article «Baudelaire et M. Faguet» est de 1910. Vingt-deux ans plus tard, relisant les Nouveaux Prétextes, Gide se déclare «content d'avoir écrit cet article». ${ }^{19}$ Il approuve donc la manière dont il avait compris la poésie de Baudelaire dans son ensemble et dont il avait jugé l'une ou l'autre de ses pièces. Ses vues sur Les Fleurs du Mal restent essentiellement identiques jusqu'à la fin, ce que nous prouve son Anthologie de la poésie française commencée avant la deuxième guerre mondiale mais achevée seulement en 1948, trois ans avant sa mort. Dans cet ouvrage Gide assigne naturellement une place d'honneur à Baudelaire qui y est représenté par 39 pièces; seul Hugo le dépasse avec 41 titres. Le choix des pièces de Baudelaire n'était pas facile; Gide toucha juste en confessant: "Il n'est pas un des poèmes des Fleurs du Mal qui ne contienne quelques vers pour l'amour desquels j'étais tenté de citer toute la pièce» (Anth., $\left.{ }^{20} 45\right)$.

19 Les Cahiers de la Petite Dame, II - Cahiers André Gide, 5, Paris, Gallimard, 1974, p. 268.

${ }_{20}$ André Gide: Anthologie de la poésie française, Paris, Gallimard ( $\mathrm{Bi}$ bliothèque de la Pléiades), 1949. 
Parmi les 39 pièces de Baudelaire recueillies dans l'Anthologie figurent les six poèmes considérés déjà dans «Baudelaire et $M$. Faguet» comme très réussis. Gide y reproduit aussi la plupart des pièces dont, à différentes occasions, il avait déjà cité des vers ou mentionné les titres. Mais il y aussi dans l'Anthologie des poèmes de Baudelaire que nous n'avons pas trouvés cités ailleurs, et pour ceux-là il n'est pas toujours aisé de distinguer les motifs qui ont poussé Gide à les inclure dans son recueil de la poésie française. Après la publication de l'Anthologie Gide se sentit satisfait «de n'avoir pas trop fait prévaloir [...] [s] on goût personnel» $(J . I I, 340)$. Mais un individualiste comme Gide, dans quelle mesure y a-t-il réussi? Parmi les poèmes de l'Anthologie non cités ailleurs il y en a, sans doute, que Gide y a mis comme typiquement baudelairiens, bien qu'ils ne soient pas toujours poétiquement parfaits: ceux, d'abord, qui rendent la vision baudelairienne de l'univers ou de la mission et de la destinée du poète (Correspondances, Bénédiction, Les plaintes 'd'un Icare) ou son ennui (une partie du Voyage, le premier et le deuxième Spleen, L'Irréparable); d'autres encore qui sont significatifs pour leurs motifs sadiques, macabres ou sataniques (Le Vampire, L'Irrémédiable, Une Martyre, Un Voyage à Cythère).

Mais il y a dans l'Anthologie quelques poèmes qui rappellent de trop près certaines situations de la vie de Gide, ses sympathies et ses penchants, pour que leur choix ne révèle pas de traces évidentes de son goût personnel. Dans le sonnet Ennemi Gide a bien pu entrevoir un souvenir des orages de sa propre jeunesse et un rapport avec ses aspirations actuelles. La Musique a dû lui rappeler les moments passés au piano, comme Les Phares, outre que par la beauté des vers, l'ont enchanté probablement comme un souvenir des heures passées aux musées. Et enfin La seroante au grand coeur, un des plus beaux poèmes de Baudelaire à cause de sa force expressive et de sa profonde humanité - est-ce que Gide pouvait le lire sans être touché par la ressemblance morale qui existait, mutatis mutandis, entre la modeste et fidèle servante Mariette, figure émouvante de l'enfance de Baudelaire, «... qui dort son sommeil sous une humble pelouse», et Anna Shackleton, gouvernante de sa mère, à qui il devait les meilleurs souvenirs de ses jeunes ans et qui quitta la vie «à sa modeste manière, si doucement et si discrètement qu'on ne s'aperçut point qu'elle mourait, mais seulement qu'elle était morte» $(J . I I, 507)$ ?

Si le choix de Gide est éloquent, les omissions aussi sont significatives. Toutes les poésies de Baudelaire qu'il avait traitées comme moins bonnes dans »Baudelaire et M. Faguet» sont exclues de l'Anthologie: non seulement Une Charogne et L'Homme et la $M e r$ mais aussi les poèmes des plus remarquables: Confession, Don Juan aux Enfers, le quatrième Spleen.

Malgré ces omissions conscientes - et d'autres encore, moins conscientes - Gide a le mérite d'avoir su distinguer parfaitement quelle place exceptionnelle Baudelaire occupe dans l'histoire de la poésie. Déjà dans l'article »Baudelaire et M. Faguet» il avait constaté que non seulement la 
poésie française mais aussi la poésie européenne ne pouvait plus être la même après Les Fleurs du Mal, parce que Baudelaire, le premier, avait fait de la «perfection secrète le but et la raison de ses poèmes» $(N . P$. 142 ), et nous savons déjà ce que signifie la "perfection secrète» dans l'esthétique gidienne. Gide dit le mot conclusif et qui résume ses réflexions antérieures sur la signification des Fleurs du Mal dans la préface de son Anthologie de la poésie française. Il affirme que la nouveauté des sujets traités n'a qu'une moindre importance. La révolution sans précédents qu'apportèrent Les Fleurs du Mal, c'est leur victoire sur le lyrisme romantique et sur la rhétorique traditionnelle ou, comme s'exprime Gide, c'est «de ne plus s'abandonner au flux lyrique, de résister à la facilité de l'inspiration', au laisser-aller rhétorique, à l'entraînement des mots, des images et des conventions surannées». Avec son recueil poétique Baudelaire eut le mérite «d'inviter l'art à maîtriser la poésie» (Anth., 10).

\section{Poozetek}

\section{ANDRÉ GIDE IN BAUDELAIROVO CVET JE ZLA}

Gide se je seznanil $z$ Baudelairovo poezijo že $\mathrm{v}$ mladih letih. V svojem prvem delu, André Walter (1891), večkrat navaja verze iz Coetje zla, in sicer tako, da jih navezuje na čustvena razpoloženja glavnega junaka, ki je po svojem bistvu mladi Gide sam. Tudi $\mathrm{v}$ zrelih letih in $\mathrm{v}$ starosti se Gide $\mathrm{v}$ različnih življenjskih položajih spominja Baudelairovih verzov. Vendar pa $\mathrm{v}$ teh dveh obdobjih pristopa $k$ Baudelairovi poeziji tudi kot kritik: njeno glavno vrednost vidi $v$ obliki, kar pa zanj ne pomeni zunanje oblikovne izdelanosti, temveč »skrito popolnost «, »muzikalnost $\ll$, ki ni le $\checkmark$ besedni zvočnosti, ampak tudi in predvsem v notranjem ritmu. Poslednjič se Gide ukvarja z Baudelairom, ko izbira pesmi za svojo Antologijo francoske poezije (1949). Njegov izbor Baudelairovih pesmi je značilen: nekatere ưvršča $v$ antologijo zaradi njihove poetične popolnosti, druge, ker so njihovi motivi tipično Baudelairovi, spet druge pa očitno zato, ker je v njih našel izražena svoja osebna doživetja in izkustva. 